\title{
Special issue: Multi-Agent Systems applications in transport
}

\author{
Frank Dignum · Juan Pavón
}

Published online: 13 May 2012

(C) Springer-Verlag 2012

Transport systems have been one of the first areas where multi-agent systems (MAS) have been applied. The agent paradigm is well suited to reflect the autonomous behaviour of the entities that constitute a transport system, where decisions are made independent by the participants, but the consequences of those decisions have a big effect on the other participants. Agents can represent both the vehicles as well as the entities in the environment that collaborate for regulation of the traffic, such as traffic lights or road signals. Using agent systems thus enables the simulation of real world situations in a natural and realistic way showing the adaptive behaviour of traffic participants as well as the collective behaviour that emerges from their interactions. Using agent-based simulations of transport systems is especially useful to explore and analyse different algorithms and strategies for its organization and management. In this sense, agent-based simulations are a valuable tool for policy making because they can show in a realistic way all possible consequences of certain decisions. For example, what will happen when people will have to pay a toll during rush hours on the big highways leading to major towns. The agent-based simulations can also facilitate the actual transport management. For example, it can help deciding whether a truck should take a city route after rush hour or rather take a detour earlier on or whether traffic lights should favour public transport at all times. The simulation allows saving expenses on implementation of transport management solutions by evaluating their impact before deployment. Finally, a MAS solution can be used, later on,

\section{F. Dignum}

Universiteit Utrecht, Utrecht, The Netherlands

e-mail: F.P.M.Dignum@uu.nl

J. Pavón ( $\square)$

Universidad Complutense Madrid, Madrid, Spain

e-mail: jpavon@fdi.ucm.es as the basis for the implementation of the actual solution and be deployed to support the regulation of the transport system. Given all these benefits of agent-based systems for analysing and controlling transport systems, it is no surprise there is a lot of active research in this area.

This special issue collects some articles that show recent applications and strategies towards transport management, with a special focus on urban transport, which is a subject of interest nowadays when considering the sustainable evolution of our cities.

With this purpose in mind, the paper by Neilla Bhouri, Flavien Balbo and Suzanne Pinson, "An Agent-Based Computational Approach for Urban Traffic Regulation", considers the impact of strategies on the use of traffic lights and signalling for improvement of the regularity of services of public transport in the presence of private vehicles. An algorithm for urban traffic strategy is implemented on an agentbased model and the simulation shows how to improve both global traffic throughput and attain regularity of the bus service in a city by appropriately controlling traffic lights at intersections. With respect to other approaches, it is interesting in the sense that it guarantees certain regularity of the bus service even under stress conditions (i.e., traffic jams). This is achieved with a distributed solution where different types of entities collaborate, e.g., buses and traffic lights, at intersections.

The decisions of the population for using public or private transportation will have a big impact on the sustainability of the transport system in urban areas. The paper by Francisco Grimaldo, Miguel Lozano, Fernando Barber and Alejandro Guerra-Hernández, "Towards a Model for Urban Mobility Social Simulation", considers urban mobility, and how different situations can emerge from the (social) decisions made by each person about how to get to work every day, e.g., by train, by car, sharing a car, etc. Using agent-based 
social simulation, in this case, enables us to simulate different human behaviours (i.e., more or less individualistic) and the impact on the possible situations. This kind of tools can be of interest for policy makers as they allow working with concepts that are close to public debate, as agents support their computational representation, in this case, the way people deliberate on social issues for using private or public transportation systems.

The paper by Ivo J. P. M. Timóteo, Miguel R. Araújo, Rosaldo J. F. Rossetti, Eugénio C. Oliveira, "Using TraSMAPI for the Assessment of Multi-Agent Traffic Management Solutions", also considers the regulation of traffic with a dynamic control of traffic lights at intersections. In this case, the paper shows how traffic lights adapt to changing traffic conditions with a simple algorithm. The interest of the paper is more methodological in the sense that it shows how to test the system through cross validation by replicating it on different simulators. This is of particular importance in situations where emergent behaviour might depend on small variations in the (order of the) interactions.

Traffic transportation systems are distributed in nature, but their control can be more or less centralized. The paper by Rutger Claes and Tom Holvoet, "Weighing Communication Overhead against Travel Time Reduction in Advanced Traffic Information Systems", considers, from the perspective of drivers, what would be the appropriate configuration of an advanced traffic information system (ATIS). It analyses the relationship between the communication overhead and travel time reduction for centralized and decentralized solutions. The paper is in this sense a good example of the use of agent-based simulation for performing this type of studies prior to implementation of infrastructures that are expensive and whose effectiveness is unknown.

The next two papers consider disaster scenarios where a proper traffic management strategy becomes even more critical, and where default solutions are not valid, due to the extraordinary circumstances.

The paper by David Handford and Alex Rogers, "An Agent-Based Social Forces Model for Driver Evacuation Behaviours", models driver behaviours during evacuation scenarios. The motivation is to create effective training environments for disaster management. In these situations, driver behaviour cannot be characterized by the usual intention to travel the quickest route between two points. Instead, people use the heuristic to mimic what other people are doing and try to follow the routes taken by other vehicles. This type of social behaviour can be analysed, for instance, with crowd models that simulate thousands of people at the same time but where the people use very simple rules for decision making. The paper shows various evacuation models. It also validates the type of driver behaviour by comparing the resulting patterns to those observed in real disasters such as the 2005 evacuation of the New Orleans area during Hurricane
Katrina. This shows the importance of an agent-based simulation approach for policy making when defining the protocols for disaster management.

Also critical in emergency situations are the mechanisms for evacuation of victims, taking into account their medical condition. These mechanisms should be both simple to use by emergency personnel, as well as robust, taking into account the special circumstances where they are applied. The paper by Estanislao Mercadal, Sergi Robles, Ramon Martí, Cormac J. Sreenan and Joan Borrell, "Double Multiagent Architecture for Dynamic Triage of Victims in Emergency Scenarios" combines a wireless sensor network, an Electronic Triage Tag, and a double MAS to make the information about victims available to the emergency coordination center at a low cost. This is achieved by using the ability of mobile agents, created with the information of each victim, to reach the emergency coordination centre leaping from handheld to handheld of the emergency personnel. The information obtained in that way is used to plan the evacuation of the victims, which is dynamically updated, as any change in any victim's medical state will be easily communicated to the ECC. The paper describes the global architecture of the system, a genetic algorithm-based approach to minimize the cost of exploring the wireless sensor network, and an optimized version of Depth-First Search to compute the path used by mobile agents to perform the desired data fusion. The paper describes the simulation results, which seem quite promising and which are currently being validated with real experimental settings.

Managing wireless communications is also relevant for transport systems, especially in cases where direct communication becomes unreliable due to a disaster. In these cases ad hoc sensor networks can be applied for providing information to the participants in the transport systems. The decentralized nature of these networks implies the use of multi-hop communication protocols in which several nodes are involved in the routing tasks. Given the dynamic nature of these networks, some mechanisms should be devised to assure system robustness on failure or malicious behaviour of some components. The paper by Laurent Vercouter and Jean-Paul Jamont, "Lightweight trusted routing for Wireless Sensor Networks", presents an adaptation of a routing protocol for wireless sensor networks that introduces trust decisions to detect and avoid sensors that exhibit an incorrect behaviour. This allows creating a network where nodes do not need to authenticate their neighbours, as they assign dynamically an estimation of trust for their neighbourhood. This solution is integrated in a Multi-Wireless Agent Communication model and validated through simulation, showing the benefits of using trust for routing under different types of malfunctioning and attacks. 